\title{
An evidence-based review of ixazomib citrate and its potential in the treatment of newly diagnosed multiple myeloma
}

This article was published in the following Dove Press journal:

OncoTargets and Therapy

29 September 2014

Number of times this article has been viewed

\section{Massimo Offidani \\ Laura Corvatta ${ }^{2}$ \\ Patrizia Caraffa' \\ Silvia Gentili' \\ Laura Maracci' \\ Pietro Leoni'}

'Hematology Department, Azienda Ospedaliero Universitaria Ospedali Riuniti di Ancona, ${ }^{2}$ Division of Medicine, Ospedale Stelluti Scala, Fabriano, Italy
Correspondence: Massimo Offidani Clinica di Ematologia, Azienda Ospedaliero-Universitaria, Ospedali Riuniti di Ancona, 7I Via Conca, Ancona 60I26, Italy Tel +39 07। 5964735 $\mathrm{Fax}+390712183448$

Email massimo.offidani@ospedaliriuniti. marche.it
Abstract: Proteasome inhibition represents one of the more important therapeutic targets in the treatment of multiple myeloma (MM), since by suppressing nuclear factor- $\mathrm{\kappa B}$ activity, which promotes myelomagenesis, it makes plasma cells susceptible to proapoptotic signals. Bortezomib, the first proteasome inhibitor approved for MM therapy, has been shown to increase response rate and improve outcome in patients with relapsed/refractory disease and in the frontline setting, particularly when combined with immunomodulatory drugs and alkylating agents. Among second-generation proteasome inhibitors, ixazomib (MLN9708) is the first oral compound to be evaluated for the treatment of MM. Ixazomib has shown improved pharmacokinetic and pharmacodynamic parameters compared with bortezomib, in addition to similar efficacy in the control of myeloma growth and prevention of bone loss. Ixazomib was found to overcome bortezomib resistance and to trigger synergistic antimyeloma activity with dexamethasone, lenalidomide, and histone deacetylase inhibitors. Phase I/II studies using ixazomib weekly or twice weekly in relapsed/refractory MM patients suggested antitumor activity of the single agent, but more promising results have been obtained with the combination of ixazomib, lenalidomide, and dexamethasone in newly diagnosed MM. Ixazomib has also been used in systemic amyloidosis as a single agent, showing important activity in this difficult-to-treat plasma-cell dyscrasia. More frequent side effects observed during administration of ixazomib were thrombocytopenia, nausea, vomiting, diarrhea, fatigue, and rash, whereas severe peripheral neuropathy was rare. Here, we review the chemical characteristics of ixazomib, as well as its mechanism of action and results from preclinical and clinical trials.

Keywords: ixazomib, MLN9708, proteasome inhibitors, multiple myeloma

\section{Introduction}

The ubiquitin-proteasome signaling pathway plays a critical role in several processes regulating cellular homeostasis and survival. It represents the main nonlysosomal route for degradation of misfolded, oxidized, or otherwise damaged protein. ${ }^{1,2}$ The proteasome is a $26 \mathrm{~S}$ multicatalytic protease that comprises $19 \mathrm{~S}$ regulatory units flanking the $20 \mathrm{~S}$ proteasome catalytic core, a barrel-shaped structure composed of two identical $\alpha$-rings and two identical $\beta$-rings (Figure 1). The two main functions of 19S units are to recognize ubiquitinated proteins and to open the $\alpha$-ring, allowing the entry of the substrate into the $20 \mathrm{~S}$ proteolytic core. ${ }^{3}$ Protein degradation via the ubiquitin-proteasome pathway occurs by a conjugation of multiple ubiquitin molecules that target the substrate; a step in which different specific enzymes (E1, E2, E3) are involved. Subsequently, the tagged protein is degraded, with the release of short peptides and free, reusable ubiquitin. ${ }^{4-6}$ The ubiquitin-proteasome pathway is able to regulate the activation of 


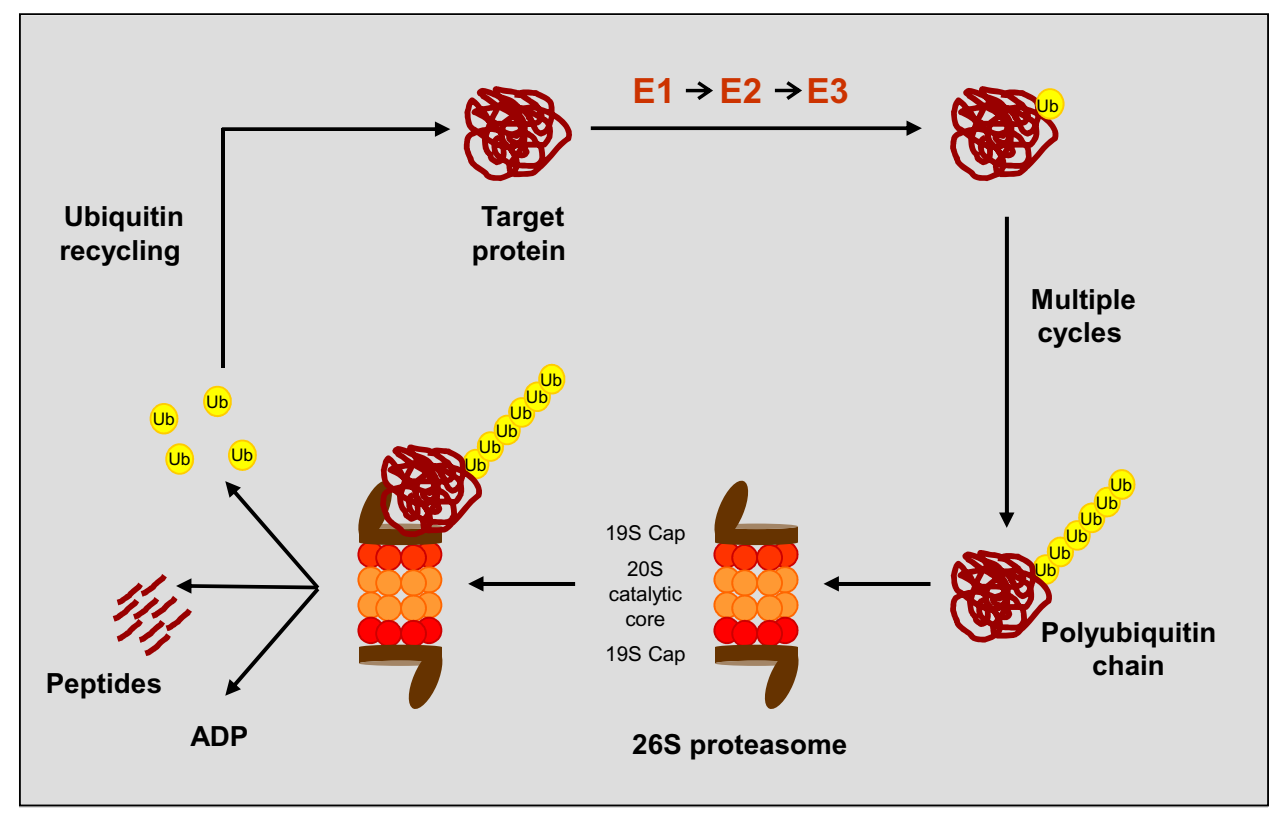

Figure I The ubiquitin proteolytic pathway.

Abbreviations: EI, ubiquitin-activating enzyme; E2, ubiquitin-conjugating enzyme; E3, ubiquitin-protein ligase; Ub, ubiquitin.

the transcriptional factor nuclear factor (NF)- $\mathrm{BB}$, which once activated translocates to the nucleus and promotes myelomagenesis by inducing the expression of growth and angiogenic factors, activating cell-cycle regulators, inhibiting apoptosis, and enhancing myeloma-cell adherence to the stroma. ${ }^{7,8}$ Multiple myeloma (MM) cells have elevated levels of NF- $\mathrm{KB}^{9,10}$ and the antimyeloma effect of proteasome inhibition is mainly related to the suppression of NF- $\mathrm{KB}$ activation, which prevents nuclear translocation and makes plasma cells more susceptible to proapoptotic signals. ${ }^{11-13}$ However, proteasome inhibitors (PIs) have also been found to act by other mechanisms: PIs interfere with tumor cellstroma cell interaction by elevation of I- $\mathrm{\kappa B}$ levels, resulting in the inhibition of angiogenic cytokines and adhesion-molecule synthesis regulated by NF- $\mathrm{KB}$; they induce apoptosis in tumor cells and the capability to restore sensitivity of tumor cells to chemotherapy through the suppression of NF- $\mathrm{KB}$-mediated cell-survival pathways; they induce degradation of cell-cycle regulatory proteins, such as cyclins and the stabilization of the p53 protein, leading to cell apoptosis; they increase the levels of cell-cycle inhibitors, resulting in $\mathrm{G}_{1} / \mathrm{S}$ cell-cycle arrest and apoptosis and activate the JNK-signaling pathway, promoting activation of caspase-3- and caspase-8-dependent cell death. ${ }^{14}$

There are three different structural classes of PIs: boronates, epoxyketones, and salinosporamides. Bortezomib, included in the group of boronates, was the first PI to be introduced in clinical practice, and its efficacy has been shown as a single agent and in combination therapies with chemotherapeutic agents or immunomodulatory drugs (IMiDs) in patients with newly diagnosed ${ }^{15-17}$ or relapsed/ refractory $^{18-21}$ MM. Among second-generation PI, carfilzomib is an epoxyketone approved in 2012 by the US Food and Drug Administration for the treatment of patients with relapsed/refractory myeloma who have received at least two prior therapies, including bortezomib and IMiDs, based on the results of a single-arm Phase II study (PX-171-003 study). ${ }^{22}$ Phase I/II studies evaluating the activity of marizomib (salinosporamide), oprozomib (epoxyketone), and delanzomib (boronate) in MM are ongoing.

\section{Chemistry, pharmacokinetics, and pharmacodynamics}

Ixazomib (MLN9708) is a second-generation boronate PI, and represents the first oral PI to be evaluated in MM clinical trials. It was selected from a broad range of smallmolecule PIs characterized by improved pharmacokinetic and pharmacodynamic profiles compared with bortezomib. Preclinical studies have shown that ixazomib is immediately hydrolyzed to MLN2238, the biologically active form, upon exposure to aqueous solution or plasma. MLN2238 is an $\mathrm{N}$-capped dipeptidyl leucine boronic acid (Figure 2) like bortezomib, and it is able to inhibit the chymotrypsin-like proteolytic ( $\beta 5)$ site of the $20 \mathrm{~S}$ proteasome, and at higher concentrations inhibit the caspase-like ( $\beta 1)$ and trypsin-like ( $\beta 2)$ proteolytic sites. ${ }^{23}$ Like bortezomib, MLN2238 also 
<smiles>CC(C)C[C@H](NC(=O)CNC(=O)c1cc(Cl)ccc1Cl)B1OC(=O)CC(CC(=O)O)(C(=O)O)O1</smiles>

B<smiles>CC(C)C[C@H](NC(=O)CNC(=O)c1cc(Cl)ccc1Cl)B(O)O</smiles>

Figure 2 Chemical structures of MLN9708 (A) and MLN2238 (B).

exerts a time-dependent reversible proteasome inhibition but the proteasome dissociation half-life $\left(t_{1 / 2}\right)$ for MLN2238 was found to be approximately six times faster than that of bortezomib ( $t_{1 / 2} 18$ minutes versus 110 minutes $){ }^{23}$

In hematologic xenograft models, MLN2238 showed a larger volume distribution at a steady state $(20.2 \mathrm{~L} / \mathrm{kg})$ compared with bortezomib $(4.3 \mathrm{~L} / \mathrm{kg})$, which allows for better drug distribution from blood to tissue compartments and leads to significant antitumor activity. ${ }^{23}$ Pharmacodynamic studies, performed in CB17 severe combined immunodeficient mice bearing human prostate (CWR22) or human lymphoma tumors (WSU-DLCL2), evaluated the degree of $20 \mathrm{~S}$ proteasome inhibition and the expression level of GADD34 protein that is upregulated in response to deoxyribonucleic acid damage, hypoxia, and energy depletion. ${ }^{24}$ Although a similar level of blood-proteasome inhibition $\left(E_{\text {max }}\right)$ between MLN2238 and bortezomib following intravenous administration was observed, the latter showed a greater blood area under the effect versus time curve value (AUE), whereas MLN2238 showed higher tumor-proteasome inhibition in both xenograft models. In particular, in a lymphoma subcutaneous xenograft model, the tumor-to-blood AUE ratios for MLN2238 and bortezomib were 2.03 and 0.26, respectively, which is consistent with an improved pharmacodynamic effect of MLN2238. Moreover, in a disseminated lymphoma model, MLN2238 was found to exert improved antitumor activity compared with bortezomib after either intermittent or continuous administration. ${ }^{23}$ Table 1 summarizes the main differences between MLN2238 and bortezomib in terms of pharmacokinetic and pharmacodynamic parameters.

Data from Phase I monotherapy studies performed on patients with hematologic malignancies and solid tumors treated with ixazomib (intravenously and orally, using
Table I Main bortezomib and MLN9708 pharmacokinetic and pharmacodynamic parameters

\begin{tabular}{lll}
\hline Parameter & Bortezomib & MLN9708 \\
\hline Subunits inhibited & $\beta 5, \beta I$ & $\beta 5$ \\
$\beta 5$ dissociation half-life (minutes) & 110 & 18 \\
Binding kinetics & Slowly reversible & Reversible \\
$C_{\text {max }}(\mathrm{ng} / \mathrm{mL})$ & 548 & 10,500 \\
$\mathrm{AUC}_{0-24 \mathrm{~h}}(\mathrm{~h} \cdot \mathrm{ng} / \mathrm{mL})$ & 4,422 & 9,660 \\
$\mathrm{Vd}^{\mathrm{L} / \mathrm{kg})}$ & 4.3 & 20.2 \\
$\mathrm{AUE}_{0-24 \mathrm{~h}}(\% \mathrm{~L} \cdot \mathrm{h})$ & $\mathrm{I}) \mathrm{I70}$ & 718 \\
Route of administration & $\mathrm{IV} / \mathrm{SC}$ & Oral//V \\
\hline
\end{tabular}

Abbreviations: $\mathrm{C}_{\max }$, maximum concentration; $\mathrm{AUC}$, area under the concentration versus time curve; $V d$, volume of distribution; $A U E$, area under the effect versus time curve; IV, intravenous; SC, subcutaneous.

a twice-weekly schedule and a weekly schedule) showed dose-linear plasma pharmacokinetics over the higher range of doses tested $\left(1-2.34 \mathrm{mg} / \mathrm{m}^{2}\right)$. Following oral administration of ixazomib capsules, the active compound MLN2388 was rapidly absorbed (time to maximum concentration $=1$ hour), and systemic exposure was found to be similar to that achieved by intravenous administration, indicating substantial bioavailability. ${ }^{25}$

\section{Mechanism of action}

Studies performed with human MM cell lines treated with different concentrations of MLN2238 for 48 hours demonstrated the ability of this compound to induce apoptosis of MM cell lines sensitive and resistant to conventional therapies. Moreover, MLN2238 was found to overcome bortezomib resistance and to trigger cytotoxicity also against plasma cells obtained from patients resistant to lenalidomide, without affecting normal peripheral blood cells. ${ }^{26}$ In human plasmacytoma xenograft mouse models, oral doses of MLN2238 induced a significant inhibition of MM tumor growth and a significant increase in survival compared with control mice. ${ }^{26}$ In MM cells, apoptosis induced by MLN2238 has been shown to be caspase-dependent, since this compound is able to activate both caspase-9 (intrinsic) and caspase- 8 (extrinsic) apoptotic pathways through the involvement of other signaling pathways, such as p53-p21, p53-NOXAPUMA, Rb-E2F, and ER stress. ${ }^{26}$ A recent study ${ }^{27}$ reported that a tumor-suppressor gene, $M I R 33 B$, which is constitutively expressed at a low level in MM cells, plays a critical role in MLN2238-induced apoptosis. In contrast with other drugs, such as dexamethasone, lenalidomide, and histone deacetylase inhibitors, MLN2238 induces the upregulation of MIR33B expression, which leads to cell death and apoptosis in MM cells mainly by blocking PIM-1 kinase activity. The function of $M I R 33 B$ as a tumor-suppressor gene in MM 
was confirmed in studies performed in subcutaneous and disseminated human MM xenograft models that showed that the overexpression of MIR33B was associated with inhibition of tumor growth and longer survival.

MLN2238 was also found to exert antiangiogenic activity both in vitro and in vivo, as confirmed by a decreased expression of angiogenic markers in tumor sections from mice treated with MLN2388. Furthermore, MLN2388 inhibits MM proliferation induced by the interaction between tumor cells and bone marrow microenvironment, and inhibits both constitutive and TNF $\alpha$-induced NF- $\kappa \mathrm{B}$ activation. ${ }^{23}$

In vitro studies demonstrated that MLN2388 triggers synergistic anti-MM activity with dexamethasone, lenalidomide, and histone deacetylase inhibitors, such as vorinostat. ${ }^{23}$ Garcia-Gomez et $\mathrm{al}^{28}$ recently reported that MLN2388 markedly inhibits in vitro osteoclastogenesis and osteoclast reabsorption through blockade of RANKL-induced $\mathrm{NF}-\kappa \mathrm{B}$ signaling, F-actin ring disruption, and decreased expression of the $\alpha_{\mathrm{v}} \beta_{3}$ integrin. Moreover, MLN2388 is able to induce differentiation of mesenchymal stem cells into osteoblasts and to enhance osteoblast function. In a disseminated MM mouse model, oral administration of MLN2388 was found to be as effective as bortezomib in the control of myeloma growth and in the prevention of bone loss. ${ }^{28}$

\section{Clinical studies}

Two Phase I studies have evaluated ixazomib administered as monotherapy in patients with relapsed and/or refractory MM. The first study, ${ }^{29}$ which included patients enrolled at six sites in the US between 2009 and 2012, was mainly aimed at determining the maximum tolerated dose (MTD) and the safety and tolerability of ixazomib. The secondary objectives of this study were to evaluate the overall response rate and pharmacokinetic parameters of ixazomib. Patients aged $\geq 18$ years, with measurable disease, Eastern Cooperative Oncology Group (ECOG) performance status of 0-2, no grade $\geq 2$ peripheral neuropathy, and at least two prior lines of therapy, including bortezomib, carfilzomib, thalidomide, lenalidomide, and corticosteroids, were enrolled in the dose-escalation phase, in which oral MLN9708 was administered on days 1,8 , and 15 of a 28-day cycle for up to 12 cycles at doses ranging from 0.24 to $3.95 \mathrm{mg} / \mathrm{m}^{2}$. The MTD $\left(2.97 \mathrm{mg} / \mathrm{m}^{2}\right)$ was used for treatment of four expansion cohorts, including patients refractory to most recent therapy, patients relapsed after bortezomib, patients relapsed after one or more therapies, including IMiDs, and patients previously treated with carfilzomib. Adverse events were graded according to the National Cancer Institute Common Terminology Criteria for Adverse Events version 3. Blood samples were taken on cycles 1 and 2 for pharmacokinetic analyses, and response was assessed using the uniform International Myeloma Working Group criteria. Sixty patients participated in the study, 32 in the dose-escalation phase, and 31 in the expansion cohorts. Overall, median age was 64 (range 40-79) years, median number of prior regimens was four (range 1-13), 77\% of patients had undergone autologous stem cell transplant (ASCT), and 72\% were refractory to the last therapy, including $18 \%$ to bortezomib. Patients received a median of two cycles (range 1-12), and at least eight cycles were administered to $18 \%$ of patients. Grade 3-4 thrombocytopenia and neutropenia occurred in $33 \%$ and $18 \%$ of patients, respectively. Thrombocytopenia was typically transient and cyclical, and only $8 \%$ of patients required platelet transfusions. Nonhematologic adverse events mainly included diarrhea $(17 \%)$, nausea $(7 \%)$, and vomiting (5\%). Only $2 \%$ of patients experienced grade 3 peripheral neuropathy, and $12 \%$ discontinued therapy due to adverse events. Regarding ixazomib activity, at least partial remission (PR) was documented in nine (18\%) of 50 patients evaluable for response, and for the ten patients achieving at least minimal response. For the patients achieving the median duration of response was 7.3 months.

In a second Phase I study, ${ }^{30}$ with objectives, inclusion, and exclusion criteria similar to the ones from the aforementioned study, ${ }^{29}$ ixazomib was administered orally on days $1,4,8$, and 11 of a 21 -day cycle for up to 12 cycles or until disease progression or unacceptable toxicity. In the dose-escalation cohort, 26 patients received ixazomib at doses ranging from $0.24 \mathrm{mg} / \mathrm{m}^{2}$ to $2 \mathrm{mg} / \mathrm{m}^{2}$, and MTD, determined as $2 \mathrm{mg} / \mathrm{m}^{2}$, was administered to 34 patients of the expansion cohorts. With regard to the whole study population (60 patients), the median age was 65 years (range 50-86 years), median number of prior therapies was four (range 1-28), 60\% had received ASCT, and $60 \%$ of patients were refractory to the last therapy, including bortezomib in $27 \%$ of them. Overall, patients were treated with a median of four cycles (range 1-39), with $18 \%$ receiving at least 12 cycles. Grade $\geq 3$ thrombocytopenia and neutropenia were reported in $37 \%$ and $17 \%$ of patients, respectively, whereas skin disorders developed in $8 \%$ of them. Of note, rash appearance, mainly on the chest, abdomen, and back, was considered a dose-dependent effect. Therapy was well tolerated, $13 \%$ of patients discontinued it, and $38 \%$ had dose reduction due to adverse events, mainly thrombocytopenia (22\%), rash $(7 \%)$, and neutropenia $(5 \%)$. 
No grade $\geq 3$ peripheral neuropathy was reported. Among the 55 patients evaluable for response, $15 \%$ achieved at least PR and $76 \%$ stable disease or better outcome. Regarding pharmacokinetic parameters, after day 11, dosing MLN2238 terminal $t_{1 / 2}$ was found to be 3.3-7.4 days, supporting twiceweekly administration. Moreover, MLN2238 plasma exposure increased proportionally with increasing dose from 0.48 to $2.23 \mathrm{mg} / \mathrm{m}^{2}$. At the 2013 American Society of Hematology meeting, Kumar et $\mathrm{al}^{31}$ reported the results of a Phase II trial of ixazomib administered as a single agent at a dose of 5.5 mg on days 1,8 , and 15 of a 28 -day cycle in patients with relapsed MM who had previously received none or fewer than six cycles of a bortezomib-based regimen. Dexamethasone (20 $\mathrm{mg}$ on the day of and day after ixazomib) was added in patients who had not achieved at least minimal response after cycle 2, at least PR after cycle 4 or with disease progression at any time. The median age of the 33 patients enrolled was 69 years, and they had received a median of two (range 1-7) prior lines of therapy, including IMiDs (88\%), bortezomib (28\%), and SCT (59\%). Grade 3-4 adverse events occurred in $56 \%$ of patients, and those more commonly observed were thrombocytopenia, fatigue, nausea, and diarrhea. No patients developed grade 3-4 peripheral neuropathy. Dexamethasone was added to ixazomib in 59\% of patients, mainly due to nonachievement of desired response. After four cycles of therapy, at least PR was obtained by $16 \%$ of patients, and this increased to $34 \%$ after the addition of dexamethasone. With a median follow-up of 7 months, overall survival (OS) at 6 months was $96 \%$. After these Phase I and II studies showed activity of the single-agent ixazomib in relapsed/refractory MM and according to important results seen with the combination of bortezomib, lenalidomide, and dexamethasone, ${ }^{17}$ ixazomib was evaluated in a triplet oral combination in newly diagnosed MM. In a Phase I/II trial by Kumar et al, ${ }^{32}$ patients aged 18 years or older with measurable disease, ECOG performance status $0-2$, and no grade $\geq 2$ peripheral neuropathy received ixazomib on days 1,8 , and 15 , lenalidomide $25 \mathrm{mg}$ on days 1-21, and dexamethasone $40 \mathrm{mg}$ on days $1,8,15$, and 22 for up to 1228 -day cycles. Induction therapy was followed by maintenance with ixazomib on the same schedule every 28 days until progression. The primary objectives of this Phase I study were safety, tolerability, MTD, and recommended Phase II dose (RP2D) whereas Phase II evaluated response rate as complete remission (CR) plus very good partial remission (VGPR), and secondarily overall response rate, time to response and progression-free survival (PFS). In the Phase I study, which enrolled 15 patients treated with ixazomib at a dose ranging from 1.68 to $3.95 \mathrm{mg} / \mathrm{m}^{2}$, MTD was found to be $2.97 \mathrm{mg} / \mathrm{m}^{2}$ and RP2D $2.23 \mathrm{mg} / \mathrm{m}^{2}$. The latter was considered to be the recommended dose, taking into account the balance between toxicity and efficacy across multiple cycles, and it was converted to a $4 \mathrm{mg}$ fixed dose based on population pharmacokinetic analysis showing that body size did not impact on AUC or maximum concentration, thus supporting a switch to a flat dosing. ${ }^{33}$ In the Phase II study, 50 patients were enrolled. Overall, median age was 66 years (range $34-86$ years), $25 \%$ of patients had unfavorable cytogenetics, and the median number of cycles administered was six (range 1-19). Moreover, 31\% of patients underwent ASCT after a median of four cycles. With regard to the safety of the triplet combination, grade 3-4 neutropenia and thrombocytopenia occurred in $9 \%$ and $6 \%$ of patients, respectively, whereas most common grade 3-4 nonhematologic side effects were rash (18\%), vomiting (8\%), fatigue $(6 \%)$, and diarrhea $(6 \%)$. Thirty-two percent of patients developed peripheral neuropathy, but grade 3 was reported in only $3 \%$ of them. Among 65 treated patients, five (8\%) discontinued treatment due to toxicity. With regard to activity, after four cycles at least PR was documented in $94 \%$ of patients (CR 19\%, VGPR 30\%, PR 45\%), and response improved after eight cycles when CR was documented in $32 \%$ of patients. In addition, $88 \%$ of patients obtaining $\mathrm{CR}$ and evaluable for minimal residual disease (MRD), assessed by multiparameter flow cytometry, were confirmed as MRD-negative. A second Phase I/II study ${ }^{34}$ conducted by Richardson et al evaluated the combination of lenalidomide and dexamethasone plus oral ixazomib given twice weekly to newly diagnosed MM patients. In the Phase I study, 14 patients aged $\geq 18$ years received 3 or $3.7 \mathrm{mg}$ of ixazomib on days 1, 4, 8, and 11, 25 $\mathrm{mg}$ of lenalidomide on days $1-14$, and dexamethasone $(20 \mathrm{mg}$ cycles $1-8,10$ mg cycles $9-16$ on days $1,2,4,5,8,911$, and 12) for up to 1621-day cycles. A maintenance treatment with ixazomib with the same schedule was planned until progression or unacceptable toxicity. Since no dose-limiting toxicities were seen in the Phase I study, the RP2D chosen in the Phase II study was $3 \mathrm{mg}$, and 50 patients were enrolled. Among 58 patients evaluable for response, $93 \%$ achieved at least PR, $67 \%$ at least VGPR, $24 \%$ CR, 14\% stringent CR, and in the Kumar study, analysis of MRD by flow cytometry in patients with CR showed achievement of MRD negativity in $82 \%$ of them. Most common grade 3 side effects were rash (18\%), pneumonia $(6 \%)$, and peripheral neuropathy $(5 \%)$, whereas no grade 4 toxicities were reported. Discontinuation of therapy due to side effects was seen in $14 \%$ of patients. Since the rate of rash and treatment-related peripheral neuropathy was lower 
with weekly administration of MLN9708, this schedule has been chosen for a Phase III ongoing trial comparing MLN9708 plus lenalidomide and dexamethasone with placebo plus lenalidomide and dexamethasone in newly diagnosed MM (NCT01850524).

In two large Phase III trials conducted by the Intergroupe Francophone du Myelome ${ }^{35}$ and the Cancer and Leukemia Group $B,{ }^{36}$ maintenance therapy with lenalidomide $10 \mathrm{mg}$ continuously post-ASCT showed a significant benefit in terms of time to progression (TTP) and PFS, and in the Cancer and Leukemia Group B trial a significant improvement in OS was also demonstrated. Based on these results, which suggest an important role of maintenance therapy post-ASCT, Shah et $\mathrm{al}^{37}$ evaluated the combination of ixazomib with lenalidomide as maintenance therapy post-ASCT in a Phase II study. The primary objective was PFS, whereas secondary end points were the incidence of secondary malignancies, the best response rate, time to progression, and time to next therapy. Twenty patients with a median age of 60 years (range 49-74 years) received 60-180 days after ASCT maintenance therapy with lenalidomide $10 \mathrm{mg}$ daily continuously and oral ixazomib $4 \mathrm{mg}$ on days 1, 8, and 15 of 28-day cycles. Grade 3-4 toxicity of this combination was shown to be very manageable, similar to that reported with lenalidomide alone, and consisted of neutropenia and thrombocytopenia occurring in seven and three patients, respectively. Severe nonhematologic toxicity was minimal, and no patients discontinued treatment due to side effects. These promising results supported the planning of a Phase III randomized trial comparing lenalidomide plus ixazomib versus lenalidomide plus placebo following ASCT.

The introduction of PIs, particularly bortezomib, has improved the outcome of patients with another plasma-cell dyscrasia, such as amyloid light-chain amyloidosis, since it was found to exert an important activity against cells secreting amyloidogenic monoclonal proteins. ${ }^{38,39}$ Patients receiving bortezomib alone ${ }^{40}$ or in combination with cyclophosphamide or melphalan ${ }^{41-43}$ achieved hematologic response rates ranging from $67 \%$ to $94 \%$, with OS at 1 year higher than $80 \%$. Based on the efficacy of bortezomib in light-chain amyloidosis, Merlini et al reported the results of a Phase I study assessing weekly ixazomib in patients with relapsed or refractory amyloidosis who had received at least one prior therapy, with cardiac biomarker stage I/II disease and measurable organ involvement. They received ixazomib at fixed doses of $4 \mathrm{mg}$ or $5.5 \mathrm{mg}$ on days 1,8 , and 15 of 28 -day cycles for up to 12 cycles. Twenty patients were enrolled with a median age of 66 years (range 54-78 years) and a median number of prior therapies of three (range 1-7). The most important grade 3-4 side effects were nausea (31\%), diarrhea

Table 2 Ongoing clinical trials of MLN9708 in multiple myeloma and amyloidosis

\begin{tabular}{|c|c|c|}
\hline Trial & Stage & Identifier \\
\hline \multicolumn{3}{|l|}{ Relapsed/refractory multiple myeloma } \\
\hline $\begin{array}{l}\text { Phase I pharmacokinetic study of oral MLN9708 plus lenalidomide and dexamethasone in adult Asian } \\
\text { patients with relapsed and/or refractory multiple myeloma }\end{array}$ & Phase I & NCT0I645930 \\
\hline Pharmacokinetic study of oral MLN9708 plus dexamethasone in relapsed/refractory multiple myeloma patients & Phase I/IB & NCT018308I6 \\
\hline MLN9708 (ixazomib) in combination with panobinostat and dexamethasone in multiple myeloma & Phase I & NCT02057640 \\
\hline Pomalidomide and dexamethasone with or without ixazomib in patients with refractory multiple myeloma & Phase I/II & NCT02004275 \\
\hline $\begin{array}{l}\text { Ixazomib plus pomalidomide and dexamethasone in patients with relapsed or relapsed/refractory } \\
\text { multiple myeloma }\end{array}$ & Phase I/II & NCT02II9468 \\
\hline $\begin{array}{l}\text { A Phase III study comparing oral MLN9708 plus lenalidomide and dexamethasone versus placebo } \\
\text { plus lenalidomide and dexamethasone in adult patients with relapsed and/or refractory multiple myeloma }\end{array}$ & Phase III & NCT0I564537 \\
\hline \multicolumn{3}{|l|}{ Newly diagnosed multiple myeloma } \\
\hline $\begin{array}{l}\text { Ixazomib, cyclophosphamide, and dexamethasone in treating patients with previously untreated } \\
\text { symptomatic multiple myeloma }\end{array}$ & Phase I/II & NCT0I8640I8 \\
\hline $\begin{array}{l}\text { Study of oral MLN9708 in combination with melphalan and prednisone in patients with newly } \\
\text { diagnosed multiple myeloma }\end{array}$ & Phase I/II & NCT0I335685 \\
\hline $\begin{array}{l}\text { Safety and efficacy study of a triplet combination of MLN9708, lenalidomide, and dexamethasone } \\
\text { in the initial management of multiple myeloma (IFM2013-06) }\end{array}$ & Phase II & NCT01936532 \\
\hline $\begin{array}{l}\text { Phase II study to evaluate the oral combination of MLN9708 with cyclophosphamide and dexamethasone } \\
\text { in patients with newly diagnosed multiple myeloma }\end{array}$ & Phase II & NCT02046070 \\
\hline $\begin{array}{l}\text { MLN9708 plus lenalidomide and dexamethasone versus placebo plus lenalidomide and dexamethasone } \\
\text { in adult patients with newly diagnosed multiple myeloma }\end{array}$ & Phase III & NCT0I850524 \\
\hline \multicolumn{3}{|l|}{ Smoldering multiple myeloma } \\
\hline MLN9708 and dexamethasone for high-risk smoldering multiple myeloma & Phase II & NCT0I660997 \\
\hline Systemic amyloidosis & Phase I & NCTOI3I8902 \\
\hline Study of oral MLN9708 in adult patients with relapsed or refractory light-chain amyloidosis & Phase III & NCT01659658 \\
\hline
\end{tabular}


( $25 \%)$, and thrombocytopenia (25\%), but no deaths related to treatment were observed. Overall, hematologic response was observed in $55 \%$ of patients ( $10 \% \mathrm{CR}$ ), and median duration of response was 7.4 months. Moreover, organ response was documented in $30 \%$ of patients. Ixazomib pharmacokinetic data were similar to those reported by other studies in MM. ${ }^{44}$ A Phase III study comparing dexamethasone plus ixazomib versus physician's choice of treatment in relapsed or refractory systemic light-chain amyloidosis is ongoing. Table 2 summarizes ongoing clinical trials evaluating ixazomib in patients with MM and amyloidosis.

\section{Conclusion}

Compared with bortezomib, ixazomib showed an improved pharmacokinetic, pharmacodynamic profile and antitumor activity in preclinical studies. Moreover, in clinical studies, its toxicity profile also appears to be superior, particularly regarding peripheral neuropathy, possibly due to the fact that it closely targets the chymotrypsin-like enzyme.

Ixazomib also exerts antimyeloma activity as a single agent in patients refractory to bortezomib, and particularly in combination with alkylating agents and IMiDs, leading to MRD negativity in a significant proportion of patients with an easy management of adverse events. However, ixazomib's strength is its administration modality: oral at a flat dose. This characteristic allows long-term administration, preserving an optimal quality of life. While confirmation of promising data of Phase I/II studies is pending from ongoing Phase III trials, ixazomib prospectively may be suitable for all oral combinations in induction, for maintenance therapy, and for smoldering MM.

\section{Disclosure}

The authors report no conflicts of interest in this work.

\section{References}

1. Ciechanover A. The ubiquitin-proteasome proteolytic pathway. Cell. 1994;79(1):13-21.

2. Ciechanover A. The ubiquitin-proteasome pathway: on protein death and cell life. EMBO J. 1998;17(24):7151-7160.

3. Groll M, Ditzel L, Löwe J, et al. Structure of 20S proteasome from yeast at 2.4 A resolution. Nature. 1997;386(6624):463-471.

4. Orlowski M, Wilk S. Catalytic activities of the 20 S proteasome, a multicatalytic proteinase complex. Arch Biochem Biophys. 2000;383(1): $1-16$.

5. Rechsteiner MC. Ubiquitin-mediated proteolysis: an ideal pathway for systems biology analysis. Adv Exp Med Biol. 2004;547:49-59.

6. Goldberg AL. Functions of the proteasome: from protein degradation and immune surveillance to cancer therapy. Biochem Soc Trans. 2007; 35(Pt 1):12-17.

7. Chauhan D, Hideshima T, Mitsiades C, Richardson P, Anderson KC. Proteasome inhibitor therapy in multiple myeloma. Mol Cancer Ther. 2005;4(4):686-692.
8. Li ZW, Chen H, Campbell RA, Bonavida B, Berenson JR. NF-kappa B in the pathogenesis and treatment of multiple myeloma. Curr Opin Hematol. 2008;15(4):391-399.

9. Chauhan D, Uchiyama H, Akbarali Y, et al. Multiple myeloma cell adhesion-induced interleukin-6 expression in bone marrow stromal cells involves activation of NF-kappa B. Blood. 1996;87(3):1104-1112.

10. Hideshima T, Chauhan D, Richardson P, et al. NF-kappa B as a therapeutic target in multiple myeloma. J Biol Chem. 2002;277(19): 16639-16647.

11. Mitsiades NJ, Mitsiades CS, Poulaki V, et al. Biological sequelae of nuclear factor- $\mathrm{\kappa B}$ blockade in multiple myeloma: therapeutic applications. Blood. 2002;99(11):4079-4086.

12. Mitsiades NJ, Mitsiades CS, Richardson P, et al. The proteasome inhibitor PS-341 potentiates sensitivity of multiple myeloma cells to conventional chemotherapeutic agents: therapeutic applications. Blood. 2003;101(6):2377-1280.

13. Rajkumar SV, Richardson PG, Hideshima T, Anderson KC. Proteasome inhibition as a novel therapeutic target in human cancer. J Clin Oncol. 2005;23(3):630-639.

14. Ruggeri B, Miknyoczki S, Dorsey B, Ai-Min H. The development and pharmacology of proteasome inhibitors for the management and treatment of cancer. Adv Pharmacol. 2009;57:91-135.

15. San Miguel JF, Schlag R, Khuageva NK, et al. Bortezomib plus melphalan and prednisone for initial treatment of multiple myeloma. N Engl J Med. 2008;359(9):906-917.

16. Cavo M, Tacchetti P, Patriarca F, et al. Bortezomib with thalidomide plus dexamethasone compared with thalidomide plus dexamethasone as induction therapy before, and consolidation therapy after, double autologous stem-cell transplantation in newly diagnosed multiple myeloma: a randomised phase 3 study. Lancet. 2010;376(9758):2075-2085.

17. Richardson PG, Weller E, Lonial S, et al. Lenalidomide, bortezomib, and dexamethasone combination therapy in patients with newly diagnosed multiple myeloma. Blood. 2010;116(5):679-686.

18. Richardson PG, Barlogie B, Berenson J, et al. A phase 2 study of bortezomib in relapsed, refractory myeloma. $N$ Engl J Med. 2003;348(26): 2609-2617.

19. Jagannath S, Barlogie B, Berenson J, et al. A phase 2 study of two doses of bortezomib in relapsed or refractory myeloma. Br J Haematol. 2004;127(2):165-172.

20. Richardson PG, Sonneveld P, Schuster MW, et al. Bortezomib or highdose dexamethasone for relapsed multiple myeloma. $N$ Engl $J$ Med. 2005;352(24):2487-2498.

21. Orlowski RZ, Nagler A, Sonneveld P, et al. Randomized phase III study of pegylated liposomal doxorubicin plus bortezomib compared with bortezomib alone in relapsed or refractory multiple myeloma: combination therapy improves time to progression. J Clin Oncol. 2007;25(25):3892-3901.

22. Siegel DS, Martin T, Wang M, et al. A phase 2 study of single-agent carfilzomib (PX-171-003-A1) in patients with relapsed and refractory multiple myeloma. Blood. 2012;120(4):2817-2825.

23. Kupperman E, Lee EC, Cao Y, et al. Evaluation of the proteasome inhibitor MLN9708 in preclinical models of human cancer. Cancer Res. 2010;70(5):1970-1980.

24. Hollander MC1, Zhan Q, Bae I, Fornace AJ Jr. Mammalian GADD34, an apoptosis- and DNA damage-inducible gene. J Biol Chem. 1977; 272(21):13731-13733.

25. Gupta N, Liu G, Berg D, Kalebic T, Gomez-Navarro J. Clinical pharmacokinetics of intravenous and oral MLN9708, an investigational proteasome inhibitor: an analysis of data from four phase 1 monotherapy studies. Poster presented at: 53rd ASH Annual Meeting and Exposition; December 10-13, 2011; San Diego, CA.

26. Chauhan D, Tian Z, Zhou B, et al. In vitro and in vivo selective antitumor activity of a novel orally bioavailable proteasome inhibitor MLN9708 against multiple myeloma cells. Clin Cancer Res. 2011;17(16): 5311-5321.

27. Tian Z, Zhao JJ, Tai YT, et al. Investigational agent MLN9708/2238 targets tumor-suppressor miR33b in MM cells. Blood. 2012;120(19): 3958-3967. 
28. Garcia-Gomez A, Quwaider D, Canavese M, et al. Preclinical activity of the oral proteasome inhibitor MLN9708 in myeloma bone disease. Clin Cancer Res. 2014:20(6):1542-1554.

29. Kumar SK, Bensinger WI, Zimmerman TM, et al. Weekly dosing of the investigational oral proteasome inhibitor ixazomib, in relapsed/ refractory multiple myeloma: results from a phase 1 study. Blood. Epub June 5, 2014.

30. Richardson PG, Baz R, Wang M, et al. Phase 1 study of twice-weekly dosing of investigational oral proteasome inhibitor ixazomib in patients with relapsed and/or refractory multiple myeloma. Blood. Epub June 11, 2014.

31. Kumar SK, Roy V, Reeder C, et al. Phase 2 trial of single agent MLN9708 in patients with relapsed multiple myeloma not refractory to bortezomib. Poster presented at: 55th ASH Annual Meeting and Exposition; December 7-10, 2013; New Orleans, LA.

32. Kumar SK, Berdeja JG, Niesvizky R, et al. A phase $1 / 2$ study of weekly MLN9708, an investigational oral proteasome inhibitor, in combination with lenalidomide and dexamethasone in patients with previously untreated multiple myeloma. Poster presented at: 54th ASH Annual Meeting and Exposition; December 8-11, 2012; Atlanta, GA.

33. Gupta N, Saleh M, Venkatakrishnan K. Flat-dosing versus BSAbased dosing for MLN9708, an investigational proteasome inhibitor: population pharmacokinetic (PK) analysis of pooled data from 4 phase-1 studies. Poster presented at: 55th ASH Annual Meeting and Exposition; December 7-10, 2013; New Orleans, LA.

34. Richardson PG, Hoffmeister CC, Rosenbaum CA, et al. Twice-weekly oral MLN9708 (ixazomib citrate), an investigational proteasome inhibitor, in combination with lenalidomide and dexamethasone in patients with newly diagnosed multiple myeloma: final phase 1 results and phase 2 data. Poster presented at: 55th ASH Annual Meeting and Exposition; December 7-10, 2013; New Orleans, LA.

35. Attal M, Lauwers-Cances V, Marit G, et al. Lenalidomide maintenance after stem-cell transplantation for multiple myeloma. $N$ Engl J Med. 2012;366(19):1782-1791
36. McCarthy PL, Owzar K, Hofmeister CC, et al. Lenalidomide after stemcell transplantation for multiple myeloma. N Engl J Med. 2012;366(19): 1770-1781.

37. Shah JJ, Baladandayuthapani V, Weber DM, et al. Phase II study of the combination of MLN9708 with lenalidomide as maintenance therapy post autologous stem cell transplant in patients with multiple myeloma. Poster presented at: 55th ASH Annual Meeting and Exposition; December 7-10, 2013; New Orleans, LA.

38. Sitia R, Palladini G, Merlini G. Bortezomib in the treatment of AL amyloidosis: targeted therapy? Haematologica. 2007;92(10): 1302-1307.

39. Oliva L, Pengo N, Palladini G, et al. Proteasome activity and stress in light chain amyloidosis. Amyloid. 2010;17 Suppl 1:OP-043.

40. Reece DE, Hegenbart U, Sanchorawala V, et al. Efficacy and safety of once-weekly and twice-weekly bortezomib in patients with relapsed systemic AL amyloidosis: results of a phase 1/2 study. Blood. 2011;118(4): 865-873.

41. Venner CP, Lane T, Foard D, et al. Cyclophosphamide, bortezomib and dexamethasone therapy in AL amyloidosis is associated with high clonal response rates and prolonged progression-free survival. Blood. 2012;119(19):4387-4390

42. Mikhael JR, Schuster SR, Jimenez-Zepeda VH, et al. Cyclophosphamidebortezomib-dexamethasone (CyBorD) produces rapid and complete hematologic response in patients with AL amyloidosis. Blood. 2012;119(19):4391-4394.

43. Palladini G, Milani P, Foli A, et al. Treatment of AL amyloidosis with bortezomib combined with alkylating agents: results from a prospective series of unselected patients. Poster presented at: 53rd ASH Annual Meeting and Exposition; December 10-13, 2011; San Diego, CA.

44. Merlini G, Sanchorawala V, Zonder JA, et al. MLN9708, a novel, investigational oral proteasome inhibitor, in patients with relapsed or refractory light-chain amyloidosis:results of a phase 1 study. Poster presented at: 54th ASH Annual Meeting and Exposition; December 8-11, 2012; Atlanta, GA.
OncoTargets and Therapy

\section{Publish your work in this journal}

OncoTargets and Therapy is an international, peer-reviewed, open access journal focusing on the pathological basis of all cancers, potential targets for therapy and treatment protocols employed to improve the management of cancer patients. The journal also focuses on the impact of management programs and new therapeutic agents and protocols on

\section{Dovepress}

patient perspectives such as quality of life, adherence and satisfaction The manuscript management system is completely online and includes a very quick and fair peer-review system, which is all easy to use. Visit http://www.dovepress.com/testimonials.php to read real quotes from published authors. 